\title{
Mapa Exploratorio de Intervenciones Psicosociales frente al Terremoto del 27 de Febrero de 2010 en la Zona Centro-Sur de Chile.
}

\author{
Exploratory Map of Psychosocial Interventions after the February 272010 Earthquake \\ in the South-Central Zone of Chile.
}

\author{
María D. Méndez, Marcelo C. Leiva, Consuelo B. Bustos, Nadia A. Ramos y Emilio Moyano-Díaz \\ Universidad de Talca, Facultad de Psicología
}

(Rec: 5 de agosto de 2010 / Acep: 21 de octubre de 2010)

\section{Resumen}

\begin{abstract}
La presente exploración tiene por objetivo elaborar un mapa de caracterización de las primeras intervenciones psicosociales realizadas en la Región del Maule en ocasión del terremoto del 27 de febrero de 2010 en Chile. Se solicitó información a 40 organizaciones que eventualmente realizaron intervenciones en la Región del Maule y a 7 informantes claves. Fueron reportadas un total de 67 intervenciones de las que un 76,4\% fueron realizadas en las comunas de Talca, Curicó y Constitución, y mayoritariamente por instituciones públicas $(50,7 \%)$ y dirigidas a todo tipo de público $(46,2 \%)$. Los tipos de intervención más comunes son las atenciones grupales $(26,8 \%)$ y talleres $(22,3 \%)$.

Una de las principales dificultades fue obtener la información desde las organizaciones contactadas. La precariedad de las organizaciones para hacer frente a la catástrofe quedó evidenciada. No obstante, el relativamente reducido número de muertos indicaría que el trabajo educativo pre-catástrofe parece haber sido eficaz.

Palabras clave: Intervención psicosocial, terremoto, catástrofe, salud mental, 27 de febrero de 2010
\end{abstract}

\begin{abstract}
This exploratory work is intended to generate a characterization map of psychosocial interventions conducted in the Maule Region after earthquake occurred in Chile in February 272010.

Information was requested from 40 organizations and seven key informants that eventually made interventions in the Maule Region. A total of 67 interventions were reported, of which $76.4 \%$ were made in the cities of Talca, Curicó and Constitución, mostly by public institutions (50.7\%), and aimed at all audiences (46.2\%). The most common types of intervention were care groups (26.8\%) and workshops (22.3\%). A major difficulty was to obtain relevant information from the contacted organizations. The precariousness of the organizations to cope with the disaster became evident. However, the relatively small number of deaths indicates that the educational work previous to the disaster seems to have been effective.

Key words: Psychosocial intervention, earthquake, disaster, mental health, february 272010.
\end{abstract}

Méndez, M. D., Leiva, M. C., Bustos, C. B., Ramos, N. A. y Moyano-Díaz, E.

Facultad de Psicología, Universidad de Talca, Talca, Chile.

Correspondencia sobre el artículo enviar a: María D. Méndez, Facultad de Psicología

Universidad de Talca, Talca, Chile. Casilla No 747 - Talca. Dirección: Av. Lircay s/n Talca. Teléfono: +56 (71) 201664; Fax: +56 (71) 201510. E- mail: dmendez@utalca.cl

El presente estudio está enmarcado en el Programa de Investigación "Calidad de Vida y Ambientes Saludables” de la Facultad de Psicología, y financiado por la Dirección de Programas de Investigación de la Universidad de Talca, Chile. 


\section{Introducción}

Una característica de Chile reconocida a nivel mundial, es que se trata de un país sísmico. El territorio chileno tiene una extensión de norte a sur de $4300 \mathrm{~km}$. y está administrativa o geopolíticamente organizado en 15 unidades territoriales denominadas regiones, que se dividen en zonas geográficas más pequeñas llamadas provincias y éstas finalmente se subdividen en comunas (BCN, 2010). Utilizando el lenguaje de los geólogos y sismólogos, el último "gran terremoto" en el mundo sucedió en Chile el día 27 de febrero de 2010 y tuvo una intensidad de $8,8^{\circ}$ Richter. Luego del sismo principal, se produjeron varios tsunamis que afectaron una extensa zona geográfica del centro sur de Chile, abarcando una longitud de $450 \mathrm{kms}$. y un ancho de 150 kms. (Barrientos, 2010). El epicentro se ubicó en la Región del Bío Bío, aproximadamente a $500 \mathrm{~km}$. de la ciudad capital del país - Santiago - , y afectó fuertemente a la región vecina hacia el norte, la Región del Maule. Esta región está compuesta por 30 comunas y 908.097 habitantes (INE, 2010).

Este último terremoto puso a prueba las capacidades y organización de la respuesta social, sanitaria y psicológica del país a tal punto que dicho evento ha sido catalogado como una catástrofe. Se entiende por "catástrofe" a un suceso negativo, imprevisto y brutal que provoca destrucción material y pérdidas humanas importantes, ocasionando un gran número de víctimas y desorganización social (Moyano Díaz \& Olivos, 1997; Fernández, Martín \& Páez, 1999). Según un informe de las Naciones Unidas de marzo de 2010, las pérdidas por daños ascienden a USD 29.662 millones en áreas de educación, vivienda, salud, vialidad, agricultura y pesca (Naciones Unidas, 2010a). Sólo en lo que respecta a vivienda, hubo 370.051 construcciones dañadas dejando alrededor de 2 millones de personas con algún grado de perjuicio en su infraestructura básica de cobijo y que significa para el Gobierno de Chile un costo aproximado en reconstrucción habitacional de USD 2.543 millones (Naciones Unidas, 2010b; Riquelme, 2010). Las víctimas fatales fueron 521 personas y 56 aún continúan desaparecidas (Ministerio del Interior-Gobierno de Chile, 2010a; 2010b).

A pesar de ser Chile uno de los países más sísmicos del mundo, un terremoto es un hecho de baja probabilidad de ocurrencia, por esta razón, es que la población tiende a actuar con apatía, sin mantener activos sistemas de afrontamiento de catástrofes realistas y efectivos. Esto tendría como efecto, según Álvarez, Chuliá y Hernando (1992), una inadecuada planificación de la respuesta de emergencia de las instituciones. En situaciones como ésta, el liderazgo local, la organización y gestión de las autoridades y responsables comunales, junto con las redes sociales y comunidades locales, cumplen una función trascendente en el abordaje de la catástrofe. La activación de las redes de ayuda mutua ha mostrado ser una vía efectiva para la búsqueda de soluciones a los problemas consecuentes (Fernández, Martín \& Páez, 1999; Campillo, Rivas, Luna y Llor, 2004). En esta línea, Martínez, Escobar, Ramírez, Panameño y Estrada (2006) y Cohen (2008), enfatizan la importancia de las redes de soporte, el acercamiento y el apoyo entre las personas como facilitadores del proceso de adaptación y recuperación de los afectados. Por lo tanto, cuando los organismos oficiales o la comunidad se encuentran vulnerados por efecto del desastre, es necesario potenciar las redes locales.

La situación de crisis vivida, caracterizada por su complejidad e incontrolabilidad, requería de una respuesta oportuna y adecuada a las necesidades locales Aguilar y Retamal (1999).

Sin embargo, se ha constatado en diversos tipos de desastres que una de las tareas más importantes como difíciles de lograr es el liderazgo y la coordinación de la ayuda. De acuerdo con la Inter-Agency Standing Comité (IASC, 2007), en situaciones de desastre es necesario establecer un único grupo coordinador de la asistencia que integre las diversas iniciativas de apoyo, que potencie a la propia comunidad afectada a fin de que ésta retenga el control de la situación, participando en la toma de decisiones para ajustar los planes de ayuda a sus necesidades y facilitar la sustentabilidad en el tiempo. A falta de un organismo único de coordinación, la gestión de la catástrofe tiende a ser más desordenada, siendo ineficiente el control y seguimiento de la misma.

Un ejemplo de coordinación, es el reportado por Tumaylle (2001), quien describe la experiencia de un proyecto de formación de voluntarios para actuar en situaciones de desastre a cargo de la Cruz Roja en Guatemala. El proyecto fue diseñado para ser desarrollado a lo largo de 10 años y tuvo como objetivo capacitar a un amplio número de voluntarios en la realización de talleres educativos, dirigidos a personas de distintas edades y diversas comunidades del país, en temas relacionados con la prevención de desastres, cuidados sanitarios y desarrollo de microproyectos para la solución de los problemas derivados de la emergencia.

Una iniciativa similar a raíz del terremoto de este año en la Región del Maule, ha sido la conformación de una red de profesionales, representantes de organizaciones públicas y privadas y personas voluntarias, para actuar colaborativamente en la etapa post terremoto. Esta red ha servido de plataforma para desarrollar actividades tales como: identificación de necesidades de intervención psicosocial, realización de talleres psicoeducativos, formación de facilitadores en atención de necesidades emocionales derivadas del evento catastrófico e investigación sobre los efectos psicosociales del terremoto (Red de Acción Psicosocial, 2010). La idea de constituir una red surgió al coincidir o encontrarse los distintos profesionales en las diversas actividades de apoyo a la comunidad y capacitación realizadas entre marzo y abril de 2010 en diferentes organizaciones de las comunas de la región. Las conversaciones surgidas en el 
terreno relativas a la catástrofe y de cómo ayudar a mitigar sus efectos psicológicos y materiales sobre las personas, hicieron que la Facultad de Psicología de la Universidad de Talca invitara a reunirse en dependencias de ésta para dar una estructura formal a este interés en común de continuar apoyando la reconstrucción psicosocial de las comunidades y familias de la región, estableciendo relaciones de intercambio y cooperación en torno a la urgencia y la solicitud de ayuda de la población afectada. Esta organización, como lo describe García (2003), se estructura con una orgánica de red caracterizada por un equipo central y líneas funcionales y, de acuerdo a Martí y Lozares (2008), actúa con una aproximación reticular que intercambia entre sí y con la comunidad tanto contenidos fácticos o de materiales (apoyo de profesionales, material psicoeducativo, etc.) como cognitivos (talleres, charlas, etc.).

La "Guía de la IASC sobre Salud Mental y Apoyo Psicosocial en Emergencias Humanitarias y Catástrofes", sugiere el establecimiento de un único grupo coordinador de la asistencia e identifica tres tipos de respuesta ante la emergencia: preparación para la emergencia, respuesta mínima y respuesta integral, en relación a 11 funciones necesarias a desarrollar: Coordinación; diagnóstico de la situación, seguimiento y evaluación; protección de normas y derechos humanos; recursos humanos (funciones comunes); organización de la comunidad y apoyo comunitario; servicios de salud; educación; difusión de la información (funciones de salud mental y apoyo psicosocial); seguridad alimentaria y nutrición; albergues y planificación de emplazamientos; abastecimiento de agua y saneamiento (Consideraciones sociales sectoriales; IASC, 2007). En este sentido, la planificación ante una catástrofe permite disminuir el impacto de la situación adversa.

La OMS (2003) ha definido las intervenciones psicosociales como cualquier tipo de intervención que tiene efectos psicológicos y sociales (OMS, 2003) e indica que éstas varían según la fase de la emergencia. En la fase aguda, existen más tareas sociales (establecer flujo de información, distribuir alimentos y refugio, evaluación cuidadosa de cuerpos, re-establecer eventos, realización de actividades recreativas a los niños, entre otras) que psicológicas (contacto con Atención Primaria, proveer primeros auxilios psicológicos, capacitar a los trabajadores de salud). Sin embargo, en la fase denominada de reconsolidación las intervenciones psicológicas cobran más relevancia que las sociales (educación psicológica a la comunidad, educación en primeros auxilios a los trabajadores de atención primaria y líderes de la comunidad, asegurar medicación a pacientes psiquiátricos, acompañar la creación de grupos de autoayuda, trabajar en planes de desarrollo para el fortalecimiento de los programas de salud mental), ya que es esperable que las actividades sociales estén resueltas.

En este sentido, la OPS-OMS ha identificado líneas generales de acción, específicamente, para la atención de salud mental en emergencias, a saber: acciones previas de preparación, evaluación preliminar rápida de los daños y las necesidades en salud mental, atención psicosocial por personal no especializado, atención especializada, capacitación en los principios básicos de atención psicosocial, intervención en crisis y primera ayuda emocional, educación para la salud y comunicación social, coordinación intersectorial e interinstitucional, organización comunitaria, participación social y fomento de la autorresponsabilidad y sistema de registro de la información, indicadores y seguimiento (Rodríguez, Zaccarelli, y Pérez, 2006).

Todas estas guías y sugerencias de actuación ante emergencias y catástrofes plantean tareas en distintas fases y, si bien las actuaciones posibles son similares, no existe mucho consenso acerca de cuáles son las fases de la catástrofe, existiendo diferentes denominaciones y temporalidades de las mismas. La clasificación más utilizada parece ser la de la OPS (2002) que establece algunos elementos temporales de referencia, reconociendo que la duración y características de las fases dependen del tipo de evento y su magnitud. De acuerdo a la OPS (2002), las etapas del desastre serían las siguientes:

a) Fase pre-Crítica: horas o días previos. Se da a conocer información acerca del evento.

b) Fase de Crisis: evento y $72 \mathrm{hrs}$. posteriores. Generalmente hay confusión, desorganización, miedo y sentimientos de vulnerabilidad.

c) Fase Post-critica: transcurridos 30 días del evento. Existe una evaluación mas detallada de la situación y llegan los grupos de ayuda externa. Se trabaja con intervención en crisis, atención psicosocial, atención de grupos vulnerables.

d) Fase de Recuperación: posterior al primer mes. Se evidencian las secuelas sociales y de salud de las personas. Se trabaja en la recuperación definitiva. En esta fase nos encontraríamos hoy en las regiones afectadas.

Sin embargo, una clasificación que nos pareció más apropiada a las características que ha tenido esta catástrofe en particular es la propuesta por Moya et al. (2000), quien señala que cada una de las fases tiene una sintomatología asociada e intervenciones posibles. El pre-impacto es la fase que precede al evento, donde se deben tomar medidas preventivas y de mitigación, principalmente a través de la educación de la población para que tome precauciones. La fase de impacto es el momento en que se vivencia el desastre y se generan pérdidas materiales y humanas; las reacciones emocionales son intensas y la duración aproximada es de un mes (Reyes, s/f). La fase post-impacto se denomina también de recuperación, y su foco es principalmente el trabajo de reconstrucción material y social. Reyes por su parte identifica dos etapas en esta fase, la de corto plazo (uno a tres meses) y la de largo plazo (más de tres meses).

En la presente exploración y dado que este documento es escrito a más de cinco meses de acaecida la catástrofe, 
se pretende identificar cuáles han sido las intervenciones realizadas en la fase post-crítica de corto plazo (de uno a 3 meses post-catástrofe).

La revisión de las investigaciones acerca de las catástrofes en el ámbito de la salud mental muestra que éstas se han focalizado principalmente en variables y reacciones psicológicas (Bödvarsdóttir y Elklit, 2004; Ibáñez, Buck, Khatchikian y Norris, 2004; Moyano Díaz y Olivos, 1997), sintomatología de estrés post-traumático (Cairo, Dutta, Nawaz, Hashmi, Kasl, \& Bellido, 2010; Goenjian et al., 2008; Groome y Soureti, 2004; Jensen, Dyb, y Nygaard, 2009; Kun, Han, Chen \& Yao, 2009; McFarlane \& Papay, 1992) y tratamiento de estrés post-traumático (García y Mardones, 2010; Hamblen, Norris, Pietruszkiewicz, Gibson, Naturale, \& Louis, 2009; Fernández, 2008). En general, se observa que existe escasa investigación psicosocial sobre las catástrofes (Dodeler \& Tarquinio, 2008; Fullerton, Reissman, Gray, Flynn \& Ursano, 2010; Vijaykumar, Thara, John \& Chellappa, 2006) y que, la mayor parte de la misma está asociada a estrés pos-traumático, es decir, a la etapa de recuperación de la catástrofe. Si bien existe información acerca del manejo de víctimas de desastres, la gran mayoría de ésta se encuentra escrita en inglés, lo que se transforma en una barrera para países que hablan otro idioma (Figueroa, Marín y González, 2010).

La propuesta de Figueroa, Marín y González, (2010) es que la respuesta psicológica temprana correspondiente a la fase temprana de respuesta al desastre -primeros tres mesesdebiera organizarse en cinco niveles de atención: 1.- Difusión, 2.- Apoyo Social, 3.- Manejo Médico General, 4.- Manejo Psiquiátrico General y 5.- Psiquiátrico por expertos. Según Figueroa, Marín y González esto tendría como objetivo que sólo los pacientes con trastornos psiquiátricos sean objeto de psicoterapia y farmacoterapia (niveles 3 al 5), ya que la población general de afectados sólo requeriría Psico-educación y Primeros Auxilios Psicológicos (niveles 1 y 2).

Concordante con esta visión, Cohen (2008) indica que las modalidades terapéuticas de atención psicológica en catástrofes son los primeros auxilios psicológicos, la intervención en crisis, consejería, medicación, asesoramiento, educación y trabajo de grupo. Se reconoce que la intervención terapéutica temprana ayuda a reducir la desorganización psico-cognitiva y afectiva, y aumenta la capacidad de las personas para afrontar las consecuencias del desastre. Así entonces, una tarea importante es formar al personal en primeros auxilios psicológicos (OMS, 2003; IASC, 2007; Figueroa, Marín y González, 2010). Un ejemplo de ello, fue el entrenamiento de voluntarios realizado en la India tras el tsunami en 2004, donde la normalización fue vista como un primer paso importante (Vijaykumar, Thara, John, \& Chellappa, 2006). Según estos últimos autores, el aporte de los voluntarios era posible en la medida que existía una coordinación con la red de salud mental, la que debe ser accesible para una intervención apropiada.
Ha sido evidenciado en otras experiencias de catástrofe que una de las mayores dificultades posteriores es la coordinación de ayudas, ya que se tiende a duplicar éstas de diferentes modos; organismos u organizaciones llegan al lugar sin previa comunicación o sin invitación, y si bien tienen un buen propósito, llegan con donaciones y un modelo de intervención que no considera la cultura local. Otra dificultad detectada ha sido reclutar profesionales para enviarlos a tiempo a los lugares de catástrofe y entrenarlos adecuadamente (Ganesan, 2006).

Aunque los servicios de salud después de una catástrofe son rápidamente re-establecidos y existe disponibilidad y accesibilidad, se ha constatado que la mayoría de los afectados por desastres no recurre a los servicios sanitarios en busca de ayuda (Cohen, 2008; Figueroa, Marín y González, 2010). De aquí la importancia de hacer una adecuada difusión de los recursos disponibles y de utilizar un modelo de salud de tipo comunitario en donde el profesional de la salud mental vaya y trabaje in situ, en los lugares donde la comunidad vive o se encuentra (OMS, 2003; OPS, 2002; Rodríguez, Zaccarelli \& Pérez, 2006).

A raíz del terremoto del 27 de Febrero de 2010, han sido publicados algunos artículos y trabajos relativos a propuestas psicoterapéuticas (García y Mardones, 2010), o de modelo de atención (Figueroa, Marín y González, 2010), reflexiones sobre la experiencia posterior al terremoto (Reyes, 2010; O'Ryan, 2010), impacto del terremoto en los hospitales de la región del Bío Bío (Kirsch et al., 2010) y un libro para niños y niñas creado para ayudarles a procesar la experiencia del terremoto (Muñoz, 2010), siendo una excepción encontrar un artículo referido expresamente a catástrofes y terremotos escrito en Chile antes de este último gran terremoto como es el de Moyano Díaz y Olivos (1997). En éste se reporta la existencia de estudios realizados desde la perspectiva geográfica y psicólógica en Chile sobre terremotos, erupciones volcánicas, inundaciones y contaminación atmosférica, y la comparación de los efectos depresivos y de EPT producidos por el terremoto acaecido en el país en 1985 (3/03) respecto del acaecido en California (2/05/1983) contra población control (Allen, 1993). En ninguno de estos trabajos, sin embargo, ha sido reportada información sobre las acciones concretas realizadas posterior a un terremoto.

El objetivo del presente estudio es dar cuenta de las intervenciones psicosociales realizadas en la Región del Maule durante la fase de post-impacto a corto plazo (de marzo a mayo de 2010). Se trata de sistematizar y entregar información acerca de la localización de las intervenciones realizadas, grupos beneficiarios y tipos de intervención entregadas, que por una parte, permita identificar y documentar las acciones realizadas en la región afectada y, por otra, sirva de orientación, guía y re-direccionamiento de los recursos existentes -humanos y económicos- para su mejor aprovechamiento desde ahora. 


\section{Método}

\section{Diseño}

Debido a que en la revisión de la literatura no se encontró información acerca de las intervenciones llevadas a cabo después de la catástrofe, esta investigación es de carácter descriptivo y exploratorio. Mediante ésta, esperamos entregar un primer panorama en este ámbito.

El período en revisión corresponde a la Fase de PostImpacto a corto plazo (Moya et al., 2000; Reyes, s/f), que abarca los primeros 3 meses después de la catástrofe (marzo a mayo de 2010).

\section{Muestra y procedimiento}

Se identificó y solicitó formalmente (por escrito) durante el mes de junio de 2010 a 40 organizaciones públicas, privadas y ONG de la Región del Maule, información respecto de las intervenciones que hubiesen realizado a propósito de la catástrofe del 27 de febrero. Para recolectar la información se elaboró y publicó el cuestionario en una plataforma virtual, que permitiese reunir la información pertinente (tipo de intervención, tipo de organismo ejecutor, comuna de intervención, público objetivo y número de beneficiarios). Posteriormente, fue enviada por correo electrónico la carta de invitación a participar en la investigación con la dirección electrónica del sitio web para que dispusieran del cuestionario diversas organizaciones u organismos de la Región del Maule.

Con el mismo propósito, se identificó a informantes claves de algunas de las instituciones con las cuales se tenía más cercanía o vínculos de colaboración con la Facultad de Psicología de Universidad de Talca. Con este procedimiento, se recolectó información de siete informantes claves, quienes completaron la información correspondiente a las intervenciones psicosociales realizadas por sus organizaciones de pertenencia en el período de marzo a mayo de 2010 en la Región del Maule.

Esta información fue ingresada a una base de datos y finalmente fue procesada. Cabe señalar que la carta enviada a las instituciones tuvo una muy escasa respuesta, de modo que prácticamente no operó como mecanismo de información de relevancia para este estudio el cual básicamente contó en forma casi exclusiva con los datos proporcionados por los 7 informantes claves.

\section{Instrumento}

El cuestionario a través del cual se recolectó la información indagó acerca de los siguientes aspectos:

- Nombre de la Comuna de la región en que se realizó la intervención.
- Público Objetivo al que fue orientada la intervención según categorías de etapa del desarrollo humano: infantil, adolescente, adulto, adulto mayor o todo tipo de público.

- Tipo de Organismo Ejecutor: se refiere a la organización o institución que realizó la intervención. Las categorías posibles son: institución u organismo público, institución u organismo privado, ONG y voluntarios sin personalidad jurídica.

- Tipo de Intervención Psicosocial: pudiendo tratarse de alguno de los siguientes tipos: intervención individual, intervención grupal, capacitación, charla, taller, elaboración y entrega de material psicoeducativo y otro.

- Número de beneficiarios directos: corresponde a la presencia de las personas en las actividades realizadas por los organismos o cuántos o quiénes son los que en primera instancia reciben directamente la intervención post terremoto.

\section{Resultados}

Los resultados arrojan 67 intervenciones realizadas en 10 de las 30 comunas de la Región del Maule, desconociéndose la situación de eventuales intervenciones en las otras veinte comunas (ver Figura 1). De acuerdo a esta información, las intervenciones se concentran en las comunas de Talca (capital regional), Curicó (segunda en tamaño poblacional de la región), y en Constitución (afectada por Tsunami), representando el $76,4 \%$ de las intervenciones (ver Tabla 1).

El público objetivo de las intervenciones no se reparte homogéneamente, ya que en la categoría de todo tipo de público se concentra un 46,2\% de aquéllas, seguida por las intervenciones en la categoría adultos con un 44,8\%. Las intervenciones a niños y adolescentes han sido escasas y corresponden a un 9\%, no existiendo información acerca de intervenciones en adultos mayores (ver Tabla 1).

Respecto al tipo de organismo ejecutor, se identificó que $50,7 \%$ de las intervenciones en la región fueron realizadas por organismos públicos seguidos por las ONG y voluntarios sin personalidad jurídica, con un $14,9 \%$ cada una. Los organismos privados representan un 13,4\% de las intervenciones (ver Figura 2).

En relación al tipo de intervenciones psicosociales realizadas, se identificó los porcentajes que se indica por categorías: Atención Grupal 26,8\%, Taller 22,3\%, Charla 20,8\%, Capacitación 11,9\%, Material Psico-educativo 7,4\%, Atención Individual 1,5\%, Diagnóstico 1,5\%, Artístico Cultural 1,5\%, Otras Intervenciones 6,3\%. Es considerable el porcentaje de intervenciones que van dirigidas a grupos de personas en la modalidades de taller, atenciones grupales, capacitaciones, charlas, todo lo cual alcanza un $81,8 \%$ de las intervenciones realizadas en la región. 
Figura 1: Mapa de la Intervenciones Psicosociales realizadas por comuna, en la Región del Maule entre los meses de marzo a mayo de 2010.

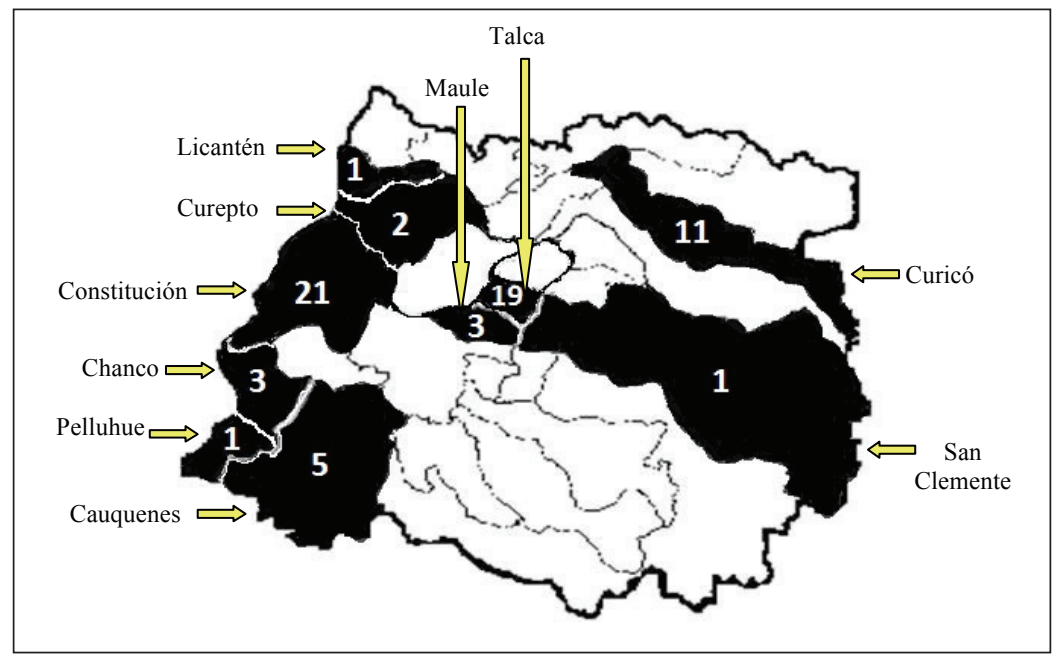

Tabla 1. Comunas de la Región del Maule y Público Objetivo en los que se han realizado intervenciones psicosociales entre marzo y mayo del 2010

\begin{tabular}{|c|c|c|c|c|c|c|}
\hline \multirow{2}{*}{$\begin{array}{l}\text { Comunas de la } \\
\text { Región del Maule }\end{array}$} & \multicolumn{4}{|c|}{ Público Objetivo de Intervención } & \multirow[b]{2}{*}{ Total } & \multirow[b]{2}{*}{ Porcentaje } \\
\hline & Niños & Adolescentes & Adultos & $\begin{array}{l}\text { Todo tipo } \\
\text { de Público }\end{array}$ & & \\
\hline Cauquenes & 2 & 0 & 0 & 3 & 5 & 7,5 \\
\hline Chanco & 0 & 0 & 0 & 3 & 3 & 4,4 \\
\hline Constitución & 3 & 0 & 12 & 6 & 21 & 31,3 \\
\hline Curepto & 0 & 0 & 0 & 2 & 2 & 3 \\
\hline Curicó & 0 & 0 & 9 & 2 & 11 & 16,5 \\
\hline Licantén & 0 & 0 & 0 & 1 & 1 & 1,5 \\
\hline Maule & 0 & 0 & 2 & 1 & 3 & 4,4 \\
\hline Pelluhue & 0 & 0 & 0 & 1 & 1 & 1,5 \\
\hline San Clemente & 0 & 0 & 0 & 1 & 1 & 1,5 \\
\hline Talca & 0 & 1 & 7 & 11 & 19 & 28,4 \\
\hline Total & 5 & 1 & 30 & 31 & 67 & 100 \\
\hline
\end{tabular}

Figura 2: Tipo de Organismo Ejecutor de Intervención Psicosocial

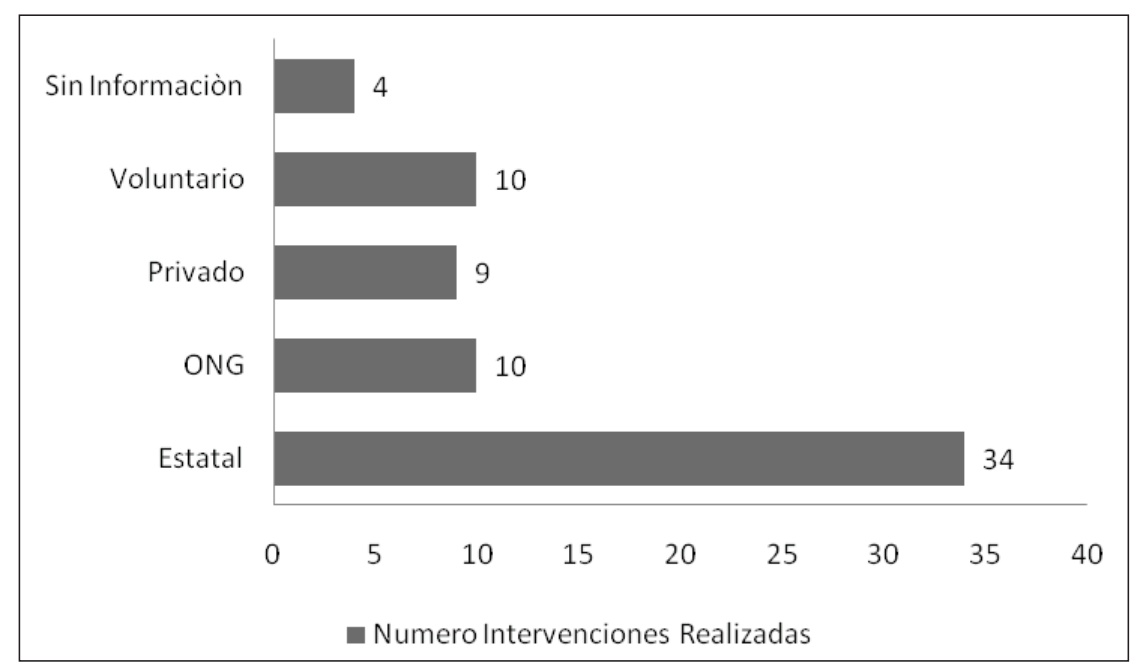


Tabla 2. Tipo Intervención Psicosocial realizada.

\begin{tabular}{lcc}
\hline Tipo intervención & Número & Porcentaje \\
\hline Artístico Cultural & 1 & 1,5 \\
Atención Grupal & 18 & 26,8 \\
Atención Individual & 1 & 1,5 \\
Capacitación & 8 & 11,9 \\
Charla & 14 & 20,8 \\
Diagnóstico & 1 & 1,5 \\
Otro & 4 & 6,3 \\
Material Psico-educativo & 5 & 7,4 \\
Taller & 15 & 22,3 \\
\hline Total & 67 & 100 \\
\hline
\end{tabular}

Tabla 3. Número de intervenciones realizadas según número de beneficiarios directos.

\begin{tabular}{|c|c|c|}
\hline Número Beneficiarios Directos & $\begin{array}{c}\text { Número Intervenciones } \\
\text { Realizadas } \\
\end{array}$ & Porcentaje \\
\hline 1 a 10 & 9 & 13,4 \\
\hline 11 a 20 & 6 & 8,9 \\
\hline 21 a 30 & 2 & 3,3 \\
\hline 31 a 40 & 3 & 4,4 \\
\hline 41 a 50 & 3 & 4,4 \\
\hline 51 a 60 & 0 & 0 \\
\hline 61 a 70 & 4 & 5,9 \\
\hline 71 a 80 & 0 & 0 \\
\hline 81 a 90 & 1 & 1,5 \\
\hline 91 a 100 & 2 & 3,3 \\
\hline Más de 100 & 12 & 17,9 \\
\hline Sin información & 25 & 37 \\
\hline Total & 67 & 100 \\
\hline
\end{tabular}

De acuerdo al número de beneficiarios directos de las intervenciones, se observa que la información de la mayoría de las intervenciones realizadas (37\%) no incluye el número de beneficiarios directos. Le siguen las intervenciones con más de 100 beneficiarios directos $(17,9 \%)$ y de 1 a 10 beneficiarios directos con un 13,4\% (ver Tabla 3).

Por último, se indagó acerca de la evaluación de las intervenciones realizadas, y sólo un 8,9\% de las intervenciones aparece habiendo realizado alguna actividad relativa a evaluación de la misma.

\section{Discusión}

Una de las principales dificultades al realizar esta investigación fue la escasa respuesta formal -escrita-de parte de las instituciones para informar acerca de las intervenciones que eventualmente hubiesen realizado. Esto podría deberse a que la tarea de sistematizar el trabajo requiere tiempo y, dada la urgencia inicial de apoyar a la población, la actividad de sistematización queda relegada a un segundo plano. Por otra parte, un organismo clave y articulador de las actividades es el Servicio de Salud Mental, que en el caso de la Región del Maule debe responder a la demanda de sus 30 comunas y casi millón de habitantes. Por la extensión geográfica y el escaso recurso humano, que pareciera haber en los servicios de salud (Ministerio de Salud, 2006), resulta comprensible -no justificable-las dificultades tanto para organizar toda la posible ayuda hacia las comunidades como para sistematizar la información rápidamente y dejarla disponible para usuarios interesados.

Una alternativa de procesamiento al respecto, es el acompañamiento y ayuda concreta que pueden realizar las universidades regionales. Estas instituciones cuentan con recursos humanos y técnicos para apoyar el diagnóstico, recopilación, intervención e investigación en este campo. Por lo tanto, una comunicación frecuente y directa entre los Servicios de Salud Mental y las Universidades se vuelve crítica al momento de gestionar eficientemente los recursos para afrontar una catástrofe.

Como ya lo han indicado la OPS (2002) y OMS (2003) las dificultades de registro y seguimiento son comunes en las situaciones de catástrofe. Por esto, una de las 
recomendaciones de acción en las diferentes fases de la catástrofe es la sistematización (IASC, 2007) y la coordinación centralizada que concentre las ayudas y supervise el trabajo de apoyo psicológico y las intervenciones psicosociales (Rodríguez, Zaccarelli, \& Pérez, 2006; Vijaykumar, Thara, John, \& Chellappa, 2006). Es decir, sistematizar la información es útil para no duplicar esfuerzos y para evaluar el impacto de las actividades posteriormente (Vijaykumar, et al., 2006). En ese sentido, este trabajo responde a dicha necesidad. Por ejemplo, después del 27/02 llegó a la Región del Maule un gran número de voluntarios quienes han intervenido sin informar ni coordinarse con ninguna institución u organismo regional o nacional. Esta situación, que ya ha sido reportada en otras catástrofes (Ganesan, 2006), no sólo impide una acción eficiente, sino también el conocer la situación real de intervención en la región. Distinto hubiese sido el panorama de existir desde un comienzo una coordinación que, armada con instrumentos como el que se presenta aquí, pudiese coordinar mejor las acciones de los voluntarios.

En relación con el número de intervenciones por comunas, de un total de 30 comunas para 10 de ellas se ha reportado intervenciones, la mayoría concentradas en 3 comunas. Además, al observar el mapa de intervenciones se aprecia que la mayoría se han realizado en comunas costeras de la región. Esto podría deberse a la exposición mediática producto del tsunami que, además del terremoto, estas comunas sufrieron. Sin embargo, esta información puede estar sesgada por los informantes claves por lo que se debe considerar con precaución.

Respecto del público objetivo, se ha podido observar que la mayoría de las intervenciones estaban dirigidas a "todo tipo de público" y "adultos" (91\%). En este sentido, las intervenciones han sido orientadas -tal y como recomiendan la OPS (2003) y la OMS (2002)- a todo el público en los primeros momentos de la catástrofe con el fin de hacer psico-educación y normalizar la sintomatología. Sin embargo, dado que las intervenciones observadas corresponden a los tres meses posteriores a la catástrofe, el porcentaje de intervención con niños y adultos mayores es escaso, ya que son poblaciones vulnerables a las cuales hay que prestar atención (OMS, 2003; OPS, 2002; IASC, 2007; Rodríguez, Zaccarelli, \& Pérez, 2006).

Otra población vulnerable y que no fue investigada aquí corresponde a la de los equipos de salud. Sería relevante contar con información al respecto, pues la literatura indica que son un grupo de riesgo dado el tipo de trabajo y el tiempo de exposición al sufrimiento, en la medida que se encuentran en la primera línea de intervención y contención de la población y porque, a su vez, ellos también han sido víctimas de la catástrofe (OPS, 2002; Rodríguez, Zaccarelli, \& Pérez, 2006; Cohen, 2008).

Por otra parte, se encontró que la mitad de las intervenciones reportadas han sido ejecutadas por organismos públicos, lo que da cuenta de la relevancia que tienen los sistemas públicos de atención cuando la sociedad chilena o el país se convulsionan por una catástrofe. No son los organismos privados los de la primera línea de afrontamiento y ayuda según esta pesquisa, sin perjuicio de lo cual existe algunas destacables o importantes iniciativas (Levantemos Chile, Chile ayuda a Chile, entre otras). Sin embargo, sería apropiado contar con información acerca de si son organizaciones locales o de otras regiones las que han intervenido en la Región del Maule. En futuras investigaciones, esto debiera ser considerado con el fin de conocer si los organismos públicos locales tienen o no la capacidad para resolver los problemas que sus catástrofes traen consigo.

Por otra parte, según la información obtenida aquí, la catástrofe puede ser también una oportunidad para el fortalecimiento del modelo de salud mental comunitaria en los servicios de salud nacionales. De hecho, la mayoría de las intervenciones se han centrado en todo tipo de público, en grupos de más de 100 personas, siendo además el tipo de intervención predominante la de atención grupal, los talleres, las capacitaciones y charlas $(81,8 \%)$. Esto concuerda con las recomendaciones de organismos internacionales respecto de la intervención inicial dirigida a la psico-educación y al desarrollo de vínculos con la comunidad (OMS, 2003; OPS, 2002; IASC, 2007; Rodríguez, et al., 2006).

Llama la atención que dentro de las actividades reportadas, sólo una corresponde a una actividad de diagnóstico. Esto es preocupante, puesto que en esta etapa de la crisis se esperaría contar con más diagnósticos acerca del estado de la población con el fin de focalizar los esfuerzos de intervención (OPS, 2002, IASC, 2007). Sin embargo, no es sorprendente encontrar tan escaso número, ya que el diagnóstico suele ser una debilidad en el abordaje del desastre (Ganesan, 2006).

Sobre el número de beneficiarios directos de las intervenciones se observa nuevamente la escasa sistematización de la información dado que el porcentaje más alto en este ámbito corresponde a la categoría "sin información". Al respecto, se hace necesario revisar los datos específicos de este grupo, para conocer si esta falta de información corresponde al mes de marzo (es decir, inmediatamente después del evento) o si es posterior, cuando es esperable tener más sistematizada la información.

Además de las dificultades en la sistematización, se observó que un bajo porcentaje de intervenciones $(8,9 \%)$ son evaluadas, probablemente porque nos encontramos en una fase temprana de acción y porque el recurso humano se encuentra abocado a la intervención de la población.

El presente estudio entrega información básica relativa a las formas, tipo y número de intervenciones realizadas en apoyo o servicio a las comunidades y personas afectadas por el terremoto del 27 de febrero a cinco meses de acaecido el evento.Al respecto, la principal limitación observada en este 
estudio se refiere al procedimiento de recolección de datos. Aparentemente, solicitar información a las instituciones mediante una carta, no es un método de recolección eficaz para reunir información en estos casos. Comparativamente, ha sido el método de informantes claves el que se ha mostrado como eficaz al respecto. Sin embargo, debido al escaso número de informantes, debemos interpretar con prudencia la información debido a los posibles sesgos. Al respecto y pensando en futuras investigaciones sobre las intervenciones post-catástrofe, recomendamos:

- Trabajar más estrechamente los vínculos con las instituciones e identificar previamente a los posibles informantes claves.

- Buscar activamente que los informantes claves formen parte del proceso de levantamiento de datos.

- Clarificar a los informantes e instituciones la utilidad e importancia de la etapa de levantamiento de la información. La razón principal es que esto permite focalizar los esfuerzos y recursos siempre escasos para intervenir.

Sin duda que a partir de la experiencia vivida en ocasión de la catástrofe del 27 de febrero, las instituciones involucradas en responder a ella han aprendido acerca de su propio funcionamiento y de su capacidad para afrontar esta situación de excepción. De hecho, tanto el país como el gobierno central sufrieron problemas dramáticos de organización y comunicación. Baste al respecto mencionar como ilustración de estas deficiencias lo documentado en la Oficina Nacional de Emergencia (ONEMI) por un video ampliamente difundido en diversos medios; la dificultad con la información emitida por el Servicio Hidrográfico y Oceanográfico de la Armada de Chile (SHOA) -tardía y cuyo contenido era ambiguo- hacia la ONEMI; la carencia de teléfonos satelitales a nivel de autoridades del gobierno central, de la ONEMI, de personal de Carabineros; la precariedad de las sedes regionales de ONEMI; la fragilidad de algunas estaciones de la Armada; la inexistencia de personal (y calificado) en la ONEMI; la ausencia de psicólogos y otros profesionales pertinentes, por mencionar los más visibles. Todo esto refleja el nivel de desarrollo alcanzado por nuestro país, al momento del terremoto y tsunami, en lo que respecta a medios y procedimientos para enfrentar un desastre natural.

Sin embargo, para solucionar estos problemas, no sólo se requiere un sistema eficiente de alerta temprana de terremotos y maremotos, sino que también organizaciones preparadas -material y profesionalmente- para enfrentar la emergencia y entregar apoyo posterior. Para esto, se requiere la profesionalización de la respuesta a la catástrofe, mediante personal debidamente entrenado que actúa coordinadamente en las áreas de la salud (física y mental), de reconstrucción y apoyo legal, dependiendo de la etapa de la emergencia. En ese sentido, un registro eficiente de información (que compatibilice rapidez y rigurosidad) acerca de las intervenciones realizadas o por realizar, es una acción irrenunciable.

\section{Referencias}

Aguilar, P., y Retamal, G. (1999). Respuesta educativa rápida en emergencias complejas: Documento de debate. Ginebra, UNICEF/OPEM

Allen, D.R. (1993). Handbook of Post Disaster Intervention. Journal of Social Behavior and Personality, 8, 353-368

Alvarez, C., Chuliá, V., y Hernando, A. (1992) Manual de asistencia sanitaria en las catástrofes. Madrid: ELA Editor.

Barrientos, S. (2010). Informe Técnico Actualizado 27 de Mayo de 2010. Servicio Sismológico, Universidad de Chile. Recuperado en julio 20, 2010 disponible en www.bcn.cl/

BCN, Biblioteca del Congreso Nacional de Chile (2010). Chile, nuestro país: División del territorio. Recuperado en julio 15, 2010 disponible en www.bcn.cl/

Bödvarsdóttir, I., \& Elklit, A. (2004). Psychological reactions in Icelandic earthquake survivors. Scandinavian Journal of Psychology, 45, 3-13.

Cairo, J., Dutta, S., Nawaz, H., Hashmi, S., Kasl, S., \& Bellido, E. (2010). The prevalence of posttraumatic stress disorder among adult earthquake survivors in Peru. Disaster Med Public Health Preparedness, 4, 39-46.

Campillo, M., Rivas, A., Luna, A., y Llor, B. (2004). ¿Qué pueden hacer los psicólogos en una situación de desastre? Enfermería global. Revista Electrónica Semestral de Enfermería. Recuperado en julio 22, 2010 disponible en www.um.es/eglobal/

Cohen, R. (2008). Lecciones aprendidas durante desastres naturales: 1970-2007. Revista Peruana de Medicina Experimental y Salud Pública, 25, 109-117.

Dodeler, V., \& Tarquinio, C. (2008). Evaluation à long terme de l'impact psychologique et social des affaissements miniers d'Auboué sur les sinistrés ou populations déplacées. Pratiques Psychologiques, 14, 491-504.

Fernández, I. (2008). EMDR after a critical incident: treatment of a tsunami survivor with acute posttraumatic stress disorder. Journal of EMDR Practice and research, 2, 156-159.

Fernández, I., Martín, C., \& Páez, D. (1999). Emociones y conductas colectivas en catástrofes: ansiedad y rumor, miedo y conductas de pánico. En J. Apalategui (Ed.), La anticipación de la Sociedad. Psicología Social de los movimientos sociales. Valencia: Promolibro.

Figueroa, R., Marín, H., \& González, M. (2010). Apoyo psicológico en desastres: Propuesta de un modelo de atención basado en revisiones sistemáticas y metaanálisis. Revista Médica de Chile, 138, 143-51.

Fullerton, C., Reissman, D., Gray, C., Flynn, B., \& Ursano, R. (2010). Earthquake Response and Psychosocial Health Outcomes: Applying Lessons From Integrating Systems of Care and Recovery to Haiti Disaster. Medicine and Public Health Preparedness, 4, 15-17.

Ganesan, M. (2006). Psychosocial response to disasters-some concerns. International Review Psychiatry, 18, 241-247.

García, F., \& Mardones, R. (2010). Prevención de trastorno de estrés postraumático en supervivientes del terremoto de Chile febrero de 2010: Una propuesta de intervención narrativa. Terapia Psicológica, 28, 85-93.

García, M. (2003) Estructuras organizacionales. En F. Gil y C. Alcover (Eds), Introducción a la Psicología de las Organizaciones. Madrid: Alianza.

Goenjian, A., Noble, E., Walling, D., Goenjuian, H., Karayan, I., Ritchie, T., \& Bailey, J. (2008). Heritabilities of symptoms of posttraumatic stress disorder, anxiety, and depression in earthquake exposed Armenian families. Psychiatric Genetics, 18, 261-266.

Groome, D., \& Soureti, A. (2004). Post-traumatic stress disorder and anxiety symptoms in children exposed to the 1999 Greek earthquake. British Journal of Psychology, 95, 387-397.

Hamblen, J., Norris, F., Pietruszkiewicz, S., Gibson, L., Naturale, A., \& Louis, C. (2009). Cognitive Behavioral Therapy for Postdisaster Distress: A community based treatment program for survivors of Hurricane Katrina. Administration and Policy in Mental Health and Mental Health, 36, 206-214. 
IASC, Inter-Agency Standing Comité. (2007). Guía del IASC sobre salud Mental y Apoyo Psicosocial en Emergencias Humanitarias y Catástrofes. Ginebra: Inter-Agency Standing Comittee (IASC).

Ibáñez, G., Buck, C., Khatchikian, N., \& Norris, F. H. (2004). Qualitative analysis of coping strategies among mexican disaster survivors. Anxiety, Stress \& Coping: An International Journal, 17, 69-85.

INE, Instituto Nacional de Estadística (2010). Proyecciones de población en Estadísticas demográficas y vitales. Recuperado en julio 22, 2010 disponible en www.ine.cl

Jensen, T., Dyb, G., \& Nygaard, E. (2009). A Longitudinal Study of Posttraumatic Stress Reactions in Norwegian Children and Adolescents Exposed to the 2004 Tsunami. Archives of Pediatr Adolescent Medicine., 163, 856-861.

Kun, P., Han, S., Chen, X., \& Yao, L. (2009). Prevalence of post-traumatic stress disorder in Sichuan Province, China after the 2008 Wenchuan earthquake. Depression and Anxiety, 26, 1134-1140.

Martínez, O., Escobar, D., Ramírez, J., Panameño, O., y Estrada, S. (2006) Manual de intervención psicosocial en situaciones de desastres. El Salvador. CIDEP. Recuperado en julio 24, 2010 disponible en http:// www.cidepelsalvador.org/documentos/manual.pdf

Martí, J., y Lozares, C. (2008). Redes organizativas locales y capital social: enfoques complementarios desde el análisis de redes sociales. Portularia, 8, 23-39.

McFarlane, A., \& Papay, P. (1992). Multiple Diagnoses in Posttraumatic Stress Disorder in the Victims of a Natural Disaster. The journal of nervous and mental disease, 180, 498-504.

Ministerio del Interior-Gobierno de Chile (2010a). Fallecidos confirmados con RUT y certificado de defunción de registro civil (actualizada al 15/04/2010). Recuperado en julio 14, 2010 disponible en http://www. interior.gov.cl/

Ministerio del Interior-Gobierno de Chile (2010b). Denuncias por presunta desgracia relacionada con el terremoto y maremoto del 27 de febrero (actualizada al 15/04/2010). Recuperado en julio 14, 2010 disponible en http://www.interior.gov.cl/

Ministerio de Salud (2006). Informe WHO-AIMS sobre sistema de salud mental en Chile. Santiago, Chile: Ministerio de Salud.

Moya, M., Gupio, G., Vilchez, L., Tello, D., Becerra, L., Marchena, A., Chucon, V., \& San Martín, J. (2000). Salud Mental en el afronte de desastres. Perú: Instituto Nacional de Salud Mental "Honorio Delgado Hideyo Noguchi".

Moyano Díaz, E., y Olivos, P. (1997) Psicología y desastres ambientales en Chile. Red de estudios sociales en Prevención de desastres en América Latina: Desastres y Sociedad, 8, 78-95.
Muñoz, B. (2010). Bruno y Violeta sobreviven al terremoto. Recuperado en julio 18, 2010 disponible en: http://www.archive.org/details/BrunoYVioletaSobrevivenAlTerremoto

Naciones Unidas (2010a). Chile Terremoto. Informe $n^{\circ}$ 9, 26 de marzo de 2010. Recuperado en julio 24, 2010 disponible en http://redhum.org/ archivos/pdf/ID_7384_VV_Redhum-CL-Informe_de_situacion_No._9Terremoto-SNU-20100326.pdf

Naciones Unidas (2010b). Chile Terremoto. Informe $n^{\circ}$ 10, 30 de marzo de 2010. Recuperado en julio 24, 2010 disponible en http://redhum.org/archivos/pdf/ID_7397_VV_Redhum-CL-Informe_de_situacion_No._10Terremoto-SNU-20100330.pdf

OPS, Organización Panamericana de Salud. (2002). Protección de la salud mental en situaciones de desastres y emergencias. Washington, DC.: OPS/OMS.

OMS, Organización Mundial de la Salud (2003). La salud Mental en las Emergencias. Ginebra: OMS.

O'Ryan, M. (2010). Reflexiones y sentimientos desde un equipo de voluntarios del Ministerio de Salud y el Colegio Médico de Chile, después del terremoto reciente. Revista Médica de Chile, 138, 270-273.

Red de Acción Psicosocial (2010). Red de Acción Psicosocial ante Catástrofes de la Región del Maule. Recuperado en julio 15, 2010 disponible en http://red-accion-maule-catastrofes.blogspot.com/

Reyes, J. (s/f). Manual de Intervenciones en salud mental para la atención de personas vistimas de desastres: Guía para el personal de apoyo en desastres (PAD). OPS/OMS Honduras. Recuperado en julio 18, 2010 disponible en http://cidbimena.desastres.hn/ri-hn/pdf/spa/doc14718/ doc14718.htm

Reyes, H. (2010). Chile se pone de pie otra vez. Revista Médica de Chile, $138,267-269$.

Riquelme, C. (2010, marzo 03). El terremoto en Chile deja 795 muertos y dos millones de damnificados. Europapress. Recuperado en Marzo 7, 2010 disponible en http://www.europapress.es/internacional/ noticia-terremoto-chile-deja-795-muertos-dos-millones-damnificados-20100302225048.html

Rodríguez, J., Zaccarelli, M., \& Pérez, R. (2006). Guía practica de la salud mental en desastres. Washington, DC.: OPS/OMS.

Tumaylle, C. (2001). Movilización de voluntarios para reducir vulnerabilidades comunitarias: repaso del programa de educación comunitaria para la prevención de desastres de la Cruz Roja Guatemalteca. Recuperado en julio 20, 2010 disponible en www.disaster-info.net/

Vijaykumar, L., Thara, R., John, S., \& Chellappa, S. (2006). Psychosocial interventions after tsunami in Tamil Nadu, India. International Review of Psychiatry, 18, 225-231. 